\title{
First record of four Alysiinae species (Hymenoptera, Braconidae) from Brazil
}

\author{
Arouca, RG. * and Penteado-Dias, AM. \\ Departamento de Ecologia e Biologia Evolutiva, Universidade Federal de São Carlos - UFSCar, \\ Rod. Washington Luis, Km 235, CEP 13565-905, São Carlos, SP, Brazil \\ *e-mail: raquelarouca@gmail.com \\ Received June 18, 2009 - Accepted June 26, 2009 - Distributed November 30, 2009
}

(With 6 figures)

\section{Introduction}

Studying material from decayed fruits, dung, carrion, "sweeping" vegetation, Malaise, Moericke and light traps from Brazilian localities, four Alysiinae (Hymenoptera, Braconidae) species were found: Aphaereta confusa Wharton, 1994; Idiasta (Idiasta) delicata (Papp, 1969); Gnathopleura astarte (Haliday, 1838) and G. semirufa (Brullé, 1846) specimens. All these species have Neotropical distribution, but this is a first recording for Brazil. Many species of Ichneumonoidea have been studied in several Brazilian ecosystems (Arouca and PenteadoDias, 2006; Loffredo and Penteado-Dias, 2008 a,b; Onody et al., in press a,b; Shimbori and Penteado-Dias, 2005).

Aphaereta confusa (Figures 1 and 2) has been collected only in Mexico; Idiasta (Idiasta) delicata (Figure 6) in
Argentina, Colombia, French Guiana, Panama, Peru and Venezuela; and their biology is unknown. Gnathopleura astarte (Figure 5) is known from Cuba, Puerto Rico, St. Vincent and Venezuela and G. semirufa (Figure 3 and 4) from French Guiana, Guyana, Peru and Trinidad and Tobago. Gnathopleura astarte and G. semirufa have been reared from the dipterous Calliphoridae, Muscidae and Sarcophagidae usually in association with dung and carrion (Wharton, 1984).

The information on the area of sampling, date, quantity and specimens sex are shown in Table 1 . The specimens are deposited in the collection (DCBU) of the Federal University of São Carlos, in the Department of Ecology and Evolutionary Biology, São Carlos, São Paulo, Brazil.

Table 1. Information on the new record of Aphaereta confusa, Gnathopleura astarte, G. semirufa and Idiasta (I.) delicata.

\begin{tabular}{|c|c|c|c|c|}
\hline Species & Locality & Date & Trap & Quantity \\
\hline \multirow[t]{3}{*}{ A. confusa } & Quebrangulo, ES & 11.ix.2002 & $\mathrm{a}$ & $2 \pi$ \\
\hline & Vila da Glória, SC & 17-20.x.2001 & $\mathrm{b}$ & 1 오 \\
\hline & & & total & 3 \\
\hline \multirow[t]{30}{*}{ G. astarte } & Cáceres, MT & 14.v.1952, xii.1984, 7.ii.1985 & $*$ & $2 \hat{\sigma}, 1+, 2 \hat{\sigma}$ \\
\hline & Rosário Oeste, MT & xi. 1963 & $*$ & $1 \hat{\sigma}$ \\
\hline & Mato Grosso & viii.1971 & $*$ & 1 우 \\
\hline & BR $070 \mathrm{Km} \mathrm{140,} \mathrm{MT}$ & 27.ix.1984 & $\mathrm{c}$ & 10 \\
\hline & Distrito Federal & viii.1954 & * & $10^{2}$ \\
\hline & Barueri, SP & 10.iii.1957 & $*$ & $20^{\lambda}$ \\
\hline & Ubatuba, SP & 21.x.1990 & $\mathrm{d}$ & 1 오 \\
\hline & Piraju, SP & 2.xi. 1983 & $*$ & 20 \\
\hline & Piracicaba, SP & 6.ii.1965 & $*$ & $1 \hat{\sigma}$ \\
\hline & Luís Antonio, SP & 12.iii.1993, 18.xi. 1992 & $*$ & 1 त, 1 오 \\
\hline & São Carlos, SP & 22.v-5.vi.1986 & $\mathrm{e}$ & $18 \hat{\jmath}, 41$ 우 \\
\hline & São Carlos, SP & 22.iv. 1986 & $\mathrm{e}$ & $49,30^{\pi}$ \\
\hline & São José do Barreiro, SP & 4.xi. 1965 & * & $2 \pi$ \\
\hline & Santa Maria Madalena, RJ & vii. 1960 & $*$ & 10 \\
\hline & Mangaratiba, RJ & 30.iv.1962 & $*$ & $30^{2}$ \\
\hline & Passos, MG & 21-26.vi.1962, 12-17.xi.1962, 24-31.xii.1962 & * & 1 + $, 1 \hat{\sigma}, 3 \hat{\jmath}$ \\
\hline & & vi. $1963,7-22 . v i .1963,28-31 . x .1963$ & & $1 \hat{\sigma}, 1 \hat{\sigma}, 2 \hat{\jmath}$ \\
\hline & Barbacena, MG & 14-16.ii.1962 & * & 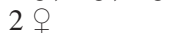 \\
\hline & Araxá, MG & 22.iii.1965 & * & 10 \\
\hline & Dourados, MS & xii. 1975 , i. 1976 & * & $1 \delta, 29$ \\
\hline & Mucuri, BA & 1-10.iii.1971 & $*$ & $1 \hat{\sigma}$ \\
\hline & Santa Teresa, ES & 27.ii.1964, 27.v.1967, 23-31.v.1967 & * & $1 \hat{\sigma}, 5 \hat{\sigma}, 1 \hat{\jmath}$ \\
\hline & Conceição da Barra, ES & 4.iv.1968, 26.iv.1967, 12.v.1969 & * & $2 \pi, 10,10$ \\
\hline & Baixo Guandu, ES & 9-15.ix.1971 & $*$ & $1 \hat{\sigma}$ \\
\hline & Curitiba, $\mathrm{PR}$ & 4.iv.1967 & $*$ & 10 \\
\hline & Guaratuba, PR & 7.ii.1965 & * & $1 \hat{\sigma}$ \\
\hline & Marumbi, PR & 18.vi.1966 & $*$ & 1 오 \\
\hline & Nova Teutônia, SC & ix.1969 & $*$ & 10 \\
\hline & Itumbiara, GO & 25.vi.2005 & $*$ & $7 \stackrel{0}{3} 3$ \\
\hline & Tucuruí, PA & 6.viii. 1980 & * & 19 \\
\hline
\end{tabular}


Table 1. Continued...

\begin{tabular}{|c|c|c|c|c|}
\hline Species & Locality & Date & Trap & Quantity \\
\hline & Pará & 27.ii.1984, 10.v.1984, 22.v.1991 & $*$ & $1 \stackrel{0}{10}, 1 \hat{0}$ \\
\hline & Imperatriz, MA & ii.1973 & $*$ & 19 \\
\hline & Ilha de Maraca, RR & 18-23.viii.1987 & * & 2 ㅇ \\
\hline & Manaus, AM & 7.viii.1969 & $*$ & 19 \\
\hline & Coari, AM & 23.xi-2.xii.1992, 11-18.v.1991 & $*$ & 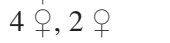 \\
\hline & & 14-25.viii.1993 & & 1 운 \\
\hline & Rondônia & 14.ix.1965 & $*$ & $1 \hat{\sigma}$ \\
\hline & & & Subtotal & 147 \\
\hline \multirow[t]{8}{*}{ G. semirufa } & Campo Grande, MS & 10.vi.1976 & $\mathrm{f}$ & 1 우 \\
\hline & Corumbá, MS & 11.xi.1993 & $*$ & $4 q$ \\
\hline & Tucuruí, PA & 4.viii.1980, 29.x-9.xi.1985 & $*$ & 1 ㅇ, 1 ㅇ \\
\hline & Pará & 28-30.i.1986 & $\mathrm{f}$ & $4 ㅇ$ \\
\hline & Cáceres, MT & 27.iii.1985 & $*$ & 19 \\
\hline & Mato Grosso & xi.1993 & $\mathrm{g}$ & 3 ㅇ, 1 ठ \\
\hline & Distrito Federal & 7.xii. 2004 & $\mathrm{f}$ & 29,20 \\
\hline & & & Subtotal & 20 \\
\hline \multirow[t]{7}{*}{ I. (I.) delicata } & São José dos Pinhais, PR & 14-21.i.1985 & $\mathrm{b}$ & 19 \\
\hline & Cáceres, MT & 26.i.1985, 30.i.1985 & b & 1 우, 1 ㅇ \\
\hline & Espírito Santo & 9-12.iv.2001 & $\mathrm{b}$ & $1 q$ \\
\hline & São Paulo & 5.v.2002 & a & 1 오 \\
\hline & Manaus, AM & 16-19.ii.2004 & $\mathrm{h}$ & $1 \delta^{\lambda}$ \\
\hline & & & Subtotal & 6 \\
\hline & & & Total & 176 \\
\hline
\end{tabular}

Notation: $\mathrm{a}=$ "Sweeping" vegetation, $\mathrm{b}=$ Malaise trap, $\mathrm{c}=$ Light trap, $\mathrm{d}=$ Moericke trap, $\mathrm{e}=$ Dung, $\mathrm{f}=$ Peckia (Squamatodes) trivittata in association with carrion, $\mathrm{g}=P$. chrysostoma in association with carrion, $\mathrm{h}=$ Decayed fruits, $*=$ No information.
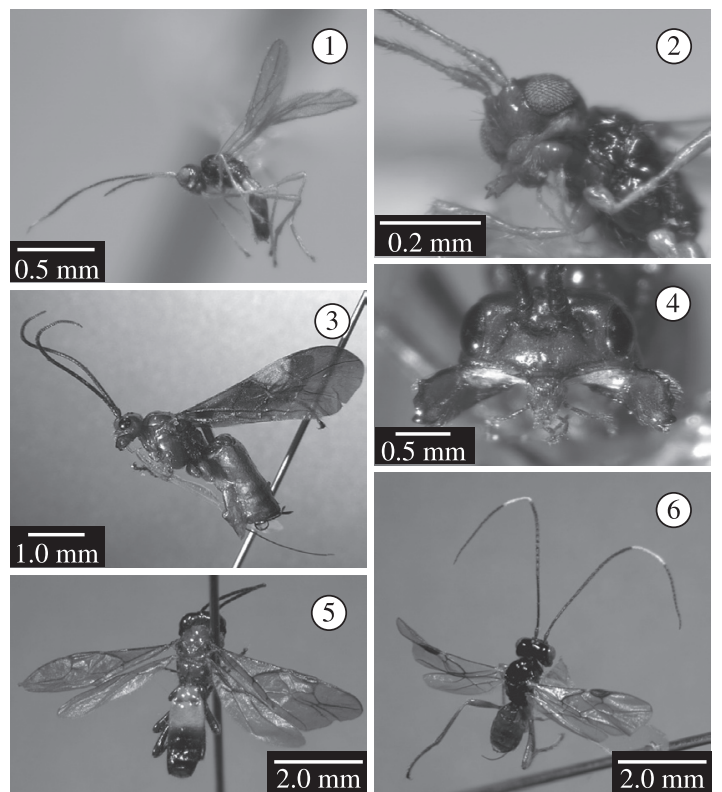

Figures 1-6. Alysiinae species. 1-2) Aphaereta confusa, $\partial^{\lambda}$. 1) habitus; 2) head showing maxillae inflated. 3-4) Gnathopleura semirufa. 3) habitus; 4) head in frontal view. 5) Gnathopleura astarte, habitus. 6) Idiasta (Idiasta) delicata, habitus.

Acknowledgements - We are grateful to the Coordenação de Aperfeiçoamento de Pessoal de Nível Superior (CAPES), Fundação de Amparo à Pesquisa do Estado de São Paulo (FAPESP), Conselho Nacional de Desenvolvimento Científico e Tecnológico (CNPq) and Instituto Nacional de Ciência e Tecnologia dos Hymenoptera Parasitóides da Região Sudeste Brasileira (INCT - HYMPAR/ Sudeste) for financial support.

\section{References}

AROUCA, RG. and PENTEADO-DIAS, AM., 2006. Contribution to systematics of neotropical species Phaenocarpa Foerster (Hymenoptera: Braconidae: Alysiinae) with description of three new species. Studies on Neotropical Fauna and Environment, vol. 41 , no. 1 , p. 33-43.

LOFFREDO, APS. and PENTEADO-DIAS, AM., 2008a. First record of Schizopyga gravenhorst (Hymenoptera, Ichneumonidae, Pimplinae) from Brazil and a description of a new species. Revista Brasileira de Biologia $=$ Brazilian Journal of Biology, vol. 68, no. 2 , p. $456-458$.

LOFFREDO, APS. and PENTEADO-DIAS, AM., 2008b. First record of Ticapimpla vilmae Gaud 1991 (Hymenoptera, Ichneumonidae, Pimplinae) from Brazil. Revista Brasileira de Biologia = Brazilian Journal of Biology, vol. 68, no. 4, p. 911.

ONODY, HC., LOFFREDO, APS. and PENTEADO-DIAS, AM., 2009. The Brachycyrtus Kriechbaumer species (Hymenoptera, Ichneumonidae, Brachycyrtinae) from Brazil. Revista Brasileira de Biologia $=$ Brazilian Journal of Biology. (No prelo).

ONODY, HC., MELO, IF., PENTEADO-DIAS, AM. and DIASFILHO, MM., 2009. New species of Eiphosoma Cresson 1865 (Hymenoptera, Ichneumonidae, Cremastinae) from Brazil. Revista Brasileira de Biologia $=$ Brazilian Journal of Biology. (No prelo).

SHIMBORI, EM. and PENTEADO-DIAS, AM., 2005. New species of Allobracon gahan, 1915 (Hymenoptera: Braconidae: Hormiinae), from Brazilian Atlantic Forest. Studies on Neotropical Fauna and Environment, vol. 42, no. 3, p. 181-202.

WHARTON, RA., 1984. Biology of the Alysiini (Hymenoptera: Braconidae), parasitoids of cyclorrhaphous Diptera. Texas Agricultural Experiment Station Technical Monograph, vol. 11, p. 39. 